\title{
In vitro pull-down assay without expression constructs
}

\author{
Harukazu Suzuki, Chihiro Ogawa, Kengo Usui, and Yoshihide Hayashizaki \\ RIKEN Genomic Sciences Center, Yokohama, Kanagawa, Japan
}

BioTechniques 37:918-920 (December 2004)

The in vitro pull-down assay is a well-established method to confirm direct binding in protein-protein interactions that was inferred from other interaction assays, such as two-hybrid analysis (1). The assay is usually carried out using glutathione-S-transferasetagged or His-tagged fusion proteins as the pull-down drivers and in vitro-translated ${ }^{35} \mathrm{~S}$-labeled proteins as probes to detect interactions. The fusion proteins and the radioactive proteins with which they interact are harvested using an affinity matrix. The co-precipitated radioactive proteins are analyzed by sodium dodecyl sulfate polyacrylamide gel electrophoresis (SDS-PAGE). The most laborious steps in the assay may be the construction of plasmids for the fusion proteins and their expression in Escherichia coli cells. In addition, fusion proteins often are expressed as insoluble forms, which further complicate their use in experiments. Here we report the development of a rapid in vitro pull-down assay that omits the use of particular expression constructs, thereby enabling evaluation of in vitro proteinprotein interactions within 1 day from sample preparation to results.

Table 1 outlines our protocol. The advantage of the assay is that it uses in vitro biotinylated proteins instead of tagged proteins as the pull-down drivers. To test the protocol, we applied it to two well-known protein-protein interactions: p53-SV40 large T antigen (2) and cFos-cJun (3). Using SDSPAGE, we successfully confirmed that radioactive p53 is co-precipitated by biotinylated simian virus 40 (SV40) large T antigen (LT) but not by biotinylated luciferase (luc; negative control), cFos, or cJun (Figure 1). We obtained the same results using biotinylated p53 and radioactive LT, and similarly, we dually confirmed the cFos-cJun interaction. In addition, we also successfully detected the previously reported self-interactions of both LT and cJun $(3,4)$. Further, we obtained almost the same results by the scintil- lation counting method (Table 2). This method is faster than SDS-PAGE, but seems sometimes less convincing because radioactively labeled high molecular weight proteins such as LT and luciferase tended to yield a higher variation of nonspecific counts than did smaller proteins. So far using our new method, we have successfully detected all six of the previously reported interactions that we tested.

The use of in vitro biotinylated proteins in pull-down assays seems to have several advantages. First, particular plasmids for the pull-down drivers need not be prepared, because most cloning vectors have bacteriophage promoters (T7, T3, or SP6 promoters) for in vitro transcription of the insert DNA and therefore the biotinylated proteins are synthesized through in vitro transcription-translation using biotin-lysine transfer RNA (tRNA)

\section{Table 1. Protocol for the Rapid In Vitro Pull-Down Assay}

1. Perform independent in vitro synthesis of biotinylated and ${ }^{35} \mathrm{~S}$-labeled proteins from the corresponding cDNA plasmids that have T7 promoter sequences upstream of the insert DNA by using the Transcend ${ }^{\mathrm{TM}}$ Biotinylated lysine-tRNA (Promega, Madison, WI, USA), ${ }^{35}$ S-methionine (Amersham Biosciences, Piscataway, NJ, USA), and TNT ${ }^{\circledR}$ T7 Quick Coupled Reticulocyte Lysate (Promega) systems and following the manufacturers' protocols.

2. Check synthesis of ${ }^{35} \mathrm{~S}$-labeled proteins by subjecting $1 \mu \mathrm{L}$ of reaction mixtures to sodium dodecyl sulfate polyacrylamide gel electrophoresis (SDS-PAGE) followed by autoradiography.

3. Mix $10 \mu \mathrm{L}$ each of biotinylated protein and ${ }^{35} \mathrm{~S}$-labeled protein (estimated concentration of $0.3-3 \mathrm{ng} / \mu \mathrm{L}$ of synthesized proteins) and incubate on ice for $1 \mathrm{~h}$.

4. Add Dynabeads ${ }^{\circledR}$ Streptavidin (Dynal Biotech LLC., Brown Deer, WI, USA) suspension [0.2 mg beads in $80 \mu \mathrm{L}$ blocking buffer ( $2 \%$ skim milk in Tris-buffered saline [TBS], $0.1 \%$ Tween ${ }^{\circledR} 20, \mathrm{pH} 8.0$ )] and incubate on a rotary shaker for $30 \mathrm{~min}$ at $4{ }^{\circ} \mathrm{C}$.

5. Isolate the beads with a magnet and wash 5 times with $150 \mu \mathrm{L}$ of ice-cold TBS, $0.1 \%$ Tween 20.

6. Subject half of the precipitated proteins to SDS-PAGE followed by autoradiography, and half to scintillation counting (optional).

Table 2. Results of Test Experiments Obtained by Scintillation Counting

\begin{tabular}{|lccccc|}
\hline & \multicolumn{5}{c|}{ Biotinylated Protein } \\
\cline { 2 - 6 } 35S-Labeled Protein & Large T & p53 & Luciferase & cFos & cJun \\
\hline Large T (LT) & 370 & 948 & 101 & 34 & 324 \\
p53 & 2483 & -62 & -115 & -72 & 174 \\
Luciferase & 1 & 187 & 270 & 70 & 135 \\
cFos & 15 & 35 & 20 & -2 & 626 \\
cJun & 16 & 23 & 36 & 344 & 101 \\
Radioactivity (counts per minute) of the other half of the reaction mixtures for which sodium dodecyl sul- \\
fate polyacrylamide gel electrophoresis (SDS-PAGE) results are shown in Figure 1. The values shown \\
$\begin{array}{l}\text { were obtained after subtraction of nonspecific counts, which were obtained by directly binding each ra- } \\
\text { dioactive protein to streptavidin beads in mock experiments in the absence of biotinylated proteins. It is } \\
\text { worth noting that radioactivity did not precisely correspond to the results of SDS-PAGE in radioactively } \\
\text { labeled LT and luciferase because of higher variation of nonspecific counts. }\end{array}$ \\
\hline
\end{tabular}




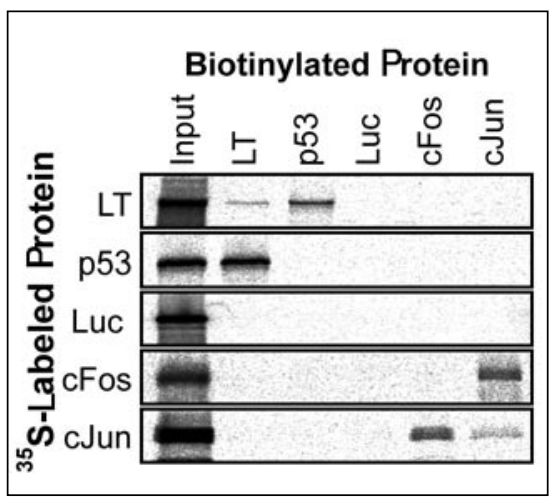

Figure 1. Sodium dodecyl sulfate polyacrylamide gel electrophoresis (SDS-PAGE) analysis of the test experiments. After the pull-down reactions, half of each reaction underwent SDSPAGE followed by autoradiography. We loaded as "input" $5 \%$ of the ${ }^{35}$ S-labeled proteins used in each reaction. LT, simian virus 40 (SV40) large $\mathrm{T}$ antigen.

(5). Second, proteins expressed by in vitro translation reactions are likely to be soluble. Third, the location of the fused tag ( $\mathrm{N}$ or $\mathrm{C}$ terminal) need not be considered in regard to interfering with the target interactions, because biotin-labeled lysine residues are incorporated randomly throughout the entire protein. Of course biotinylation of lysine residues critical for protein-protein interactions may inhibit these interactions in some proteins. Further, several studies suggest that in many cases, biotinylated proteins are functional and therefore maintain their native conformation (6-8). Therefore, like traditional pull-down assays, our method can be used to confirm in vitro protein-protein interactions.

\section{ACKNOWLEDGMENTS}

This study was supported by Core Research for Evolutional Science and Technology (CREST) of the Japan Science and Technology Agency (to Y.H.), by the Research Grants for the RIKEN Genome Exploration Research Project, the Advanced and Innovational Research Program in Life Science, and the National Project on Genome Network Analysis from the Ministry of Education, Science and Culture of Japan (MEXT) (to Y.H.), and by a Grant-inAid for Scientific Research from MEXT (to H.S.).

\section{COMPETING INTERESTS STATEMENT}

The authors declare no conflicts of interests.

\section{REFERENCES}

1.Golemis, E. (Ed.). 2002. Protein-Protein Interactions. CSH Laboratory Press, Cold Spring Harbor, NY.

2.Staufenbiel, M. and W. Deppert. 1983. Different structural systems of the nucleus are targets for SV40 large T antigen. Cell 33:173181.

3.Smeal, T., P. Angel, J. Meek, and M. Karin. 1989. Different requirements for formation of Jun:Jun and Jun:Fos complexes. Genes Dev. 3:2091-2100.

4.Bradley, M.K., J.D. Griffin, and D.M. Livingston. 1982. Relationship of oligomerization to enzymatic and DNA-binding properties of the SV40 large T antigen. Cell 28:125-134.

5.Kurzchalia, T.V., M. Wiedmann, H. Breter, W. Zimmermann, E. Bauschke, and T.A. Rapoport. 1988. tRNA-mediated labelling of proteins with biotin. A nonradioactive method for the detection of cell-free translation products. Eur. J. Biochem. 172:663-668.

6.Beckler, G.S. and R. Hurst. 1993. tRNAnscend non-radioactive detection of in vitro translation products labeled using biotinylated lysine tRNA. Promega Notes 43:24-31.

7.Sanford, J.C., J. Yu, J.Y. Pan, and M. Wessling-Resnick. 1995. GDP dissociation inhibitor serves as a cytosolic acceptor for newly synthesized and prenylated Rab5. J. Biol. Chem. 270:26904-26909.

8.de Boer, E., P. Rodriguez, E. Bonte, J. Krijgsveld, E. Katsantoni, A. Heck, F. Grosveld, and J. Strouboulis. 2003. Efficient biotinylation and single-step purification of tagged transcription factors in mammalian cells and transgenic mice. Proc. Natl. Acad. Sci. USA 100:7480-7485.

Received 24 June 2004; accepted 22 July 2004.

Address correspondence to Harukazu Suzuki, Laboratory for Genome Exploration Research Group, RIKEN Genomic Sciences Center (GSC), 1-7-22 Suehiro-cho, Tsurumi-ku, Yokohama 230-0045, Japan. e-mail: rgscerg@gsc.riken.jp 